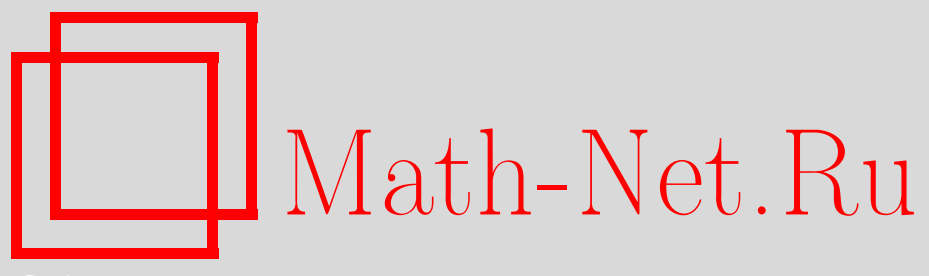

В. А. Артамонов, Л. А. Бокуть, В. В. Борисенко, Е. И. Бунина, В. М. Бухштабер, А. Э. Гутерман, Ю. Л. Ершов, М. В. Зайцев, А. Я. Канель-Белов, А. Р. Кемер, А. В. Михалев, С. П. Мищенко, С. П. Новиков, А. Ю. Ольшанский, Ю. Г. Прохоров, Ю. П. Размыслов, А. И. Шафаревич, И. В. Шестаков, Виктор Николаевич Латышев (некролог), УМН, 2022, том 77, выпуск $1,177-182$

DOI: https://doi.org/10.4213/rm9996

Использование Общероссийского математического портала Math-Net.Ru подразумевает, что вы прочитали и согласны с пользовательским соглашением http: //www . mathnet.ru/rus/agreement

Параметры загрузки:

IP : 54.198 .64 .247

26 апреля 2023 г., 14:20:03

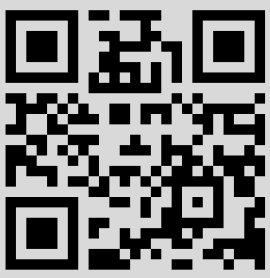




\section{Виктор Николаевич Латышев}

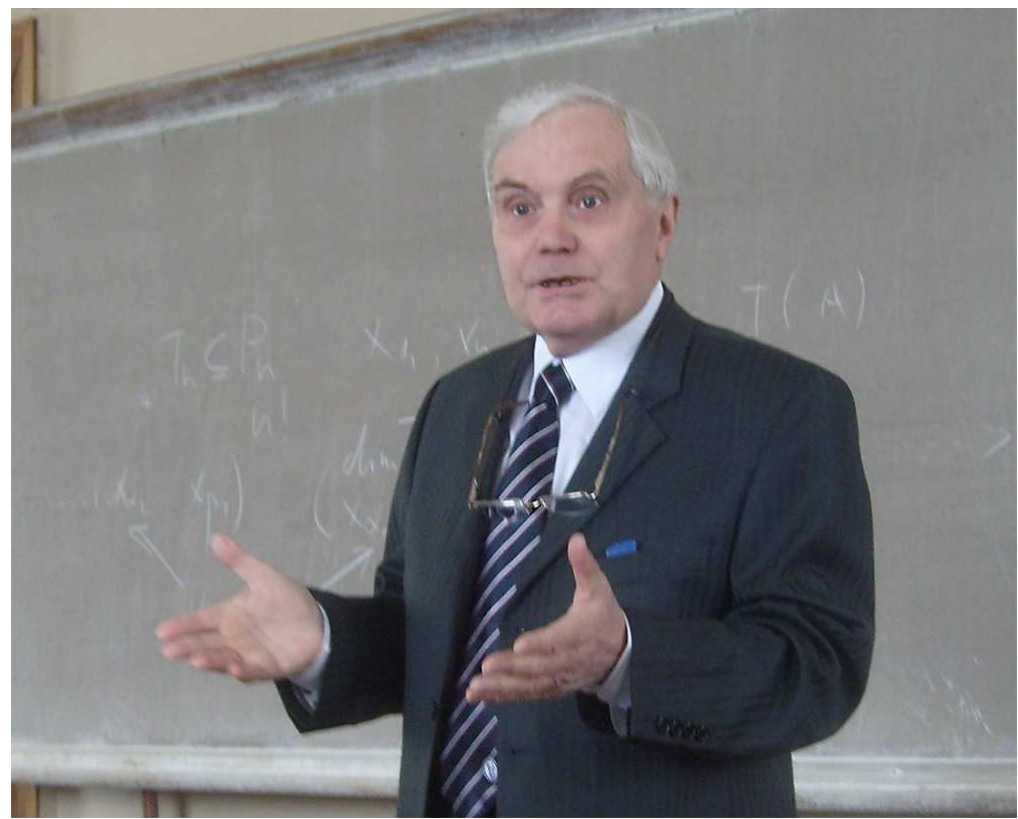

13 апреля 2020 г. ушел от нас выдающийся советский и российский математик, замечательный педагог, профессор кафедры высшей алгебры механико-математического факультета МГУ Виктор Николаевич Латышев.

В.Н. Латышев родился 9 февраля 1934 г. в Москве. Его детство прошло в подмосковном Пушкино, где он окончил школу с золотой медалью. Почти вся жизнь Виктора Николаевича была связана с Московским государственным университетом им. М. В. Ломоносова и конкретно с кафедрой высшей алгебры механико-математического факультета. Он поступил на мехмат МГУ в 1953 г., окончил его в 1958 г., став аспирантом кафедры алгебры, а в 1961 г. начал работать на кафедре. Его научным руководителем был замечательный алгебраист Анатолий Илларионович Ширшов специалистам по теории ассоциативных и лиевых колец хорошо известны результаты А. И. Ширшова, ставшие классикой в этой области: теорема Ширшова об ограниченности высот ассоциативной PI-алгебры, теорема Ширшова-Витта о свободе любой подалгебры в свободной алгебре Ли, базисы Ширшова в свободной алгебре Ли.

DOI: https://doi.org/10.4213/rm9996 
В свою очередь, А. И. Ширшов был учеником Александра Геннадьевича Куроша, возглавлявшего в то время кафедру высшей алгебры мехмата МГУ. А. И. Ширшов также тесно сотрудничал с Анатолием Ивановичем Мальцевым, возглавлявшим отдел алгебры Института математики СО АН СССР в Новосибирске. Эти ученые внесли неоценимый вклад в развитие советской школы в теории колец.

Главной областью интересов В. Н. Латышева была теория ассоциативных и лиевых колец и алгебр с полиномиальными тождествами - PІ-алгебр. Класс ассоциативных PI-алгебр обобщает классы коммутативных и конечномерных алгебр, и многие теоремы для подобных алгебр, которые не верны в общем случае, переносятся на класс PI-алгебр. В исследовании PI-алгебр можно выделить два направления: структурная теория - к ней относится, например, проблема нильпотентности радикала Джекобсона конечно порожденной РІ-алгебры, и комбинаторная теория, которая изучает свойства идеалов тождеств ( $T$-идеалов) в свободной алгебре и задаваемых ими многообразий алгебр. Работы В.Н. Латышева относятся в основном к комбинаторному направлению.

В течение долгого времени одной из центральных проблем в комбинаторной теории ассоциативных PI-алгебр была проблема Шпехта, сформулированная В. Шпехтом в 1950 г. (W. Specht, "Gesetze in Ringen. I", Math. Z., 52 (1950), 557-589): верно ли, что все тождества произвольной ассоциативной PI-алгебры следуют из конечного их числа? Эквивалентная формулировка: верно ли, что любой $T$-идеал в свободной ассоциативной алгебре конечно порожден как вполне характеристический идеал?

Проблема Шпехта оказалась сложной и потребовала для своего решения многолетних трудов многих математиков. В.Н. Латышев сделал первый, очень важный шаг в направлении ее положительного решения. Во-первых, естественно было рассматривать класс конечно порожденных алгебр - тогда почти все предполагали, что положительное решение проблемы Шпехта возможно лишь в классе конечно порожденных PI-алгебр. Во-вторых, рассмотрим наиболее простой класс РІ-алгебр - так называемые нематричные многообразия. Известно (и несложно доказывается), что для произвольной PI-алгебры $A$ все ее тождества являются степенями тождеств алгебры матриц порядка $n$; минимальное такое $n$ называется сложностью или полиномиальной степенью многообразия, порожденного алгеброй $A$. Многообразия полиномиальной сложности 1 называются нематричными, в них выполняется тождество $[x, y]^{k}=0$ для некоторого $k \in \mathbb{N}$ (через $[x, y]$ обозначен коммутатор: $[x, y]=x y-y x$ ). В конечно порожденной нематричной РІ-алгебре над полем характеристики 0 выполняется тождество вида

$$
\left[x_{1}, x_{2}\right]\left[x_{3}, x_{4}\right] \cdots\left[x_{2 n-1}, x_{2 n}\right]=0 .
$$

Именно для таких многообразий В. Н. Латышев дал положительное решение проблемы Шпехта: если в PI-алгебре над полем характеристики 0 выполняется тождество такого вида, то $T$-идеал всех ее тождеств порождается конечным набором элементов. Этот результат является основным в докторской диссертации В.Н. Латышева (1977 г.). Отметим, что фактически доказано больше, чем только шпехтовость многообразия алгебр с указанным тождеством, - дана полная классификация всех его подмногообразий. Интерес представляет также построенный для решения этой задачи базис в свободной ассоциативной алгебре, состоящий из произведений длинных коммутаторов. Раньше похожий базис был предложен в работах Шпехта, однако базис Латышева (так его теперь называют) лучше подходит для решения различных задач в нематричных многообразиях. 
В настоящее время проблема Шпехта решена положительно А.Р. Кемером для PI-алгебр над полем характеристики 0. Над полем характеристики $p$ проблема в общем случае решается отрицательно: сразу тремя математиками, А.Я. Беловым, А.В. Гришиным и В.В. Щиголевым, были построены примеры многообразий, которые невозможно задать конечным числом тождеств. В. Н. Латышев был научным руководителем В. В. Щиголева, а А. Я. Белов и А. В. Гришин постоянно участвовали в работе семинара мехмата МГУ по теории колец под руководством В. Н. Латышева и А. В. Михалева. Так что В.Н. Латышева можно считать одним из вдохновителей направления, связанного с проблемой Шпехта и теорией ассоциативных PI-алгебр.

Другой областью интересов В. Н. Латышева была алгоритмическая теория колец, возможность эффективных вычислений в кольцах путем построения стандартных базисов для конечно определенных колец и алгебр. В коммутативном случае такие базисы называются базисами Грёбнера, они всегда конечны и позволяют эффективно решать большинство задач в факторалгебрах алгебры многочленов. В некоммутативном случае стандартные базисы могут быть бесконечными, тем не менее в ряде случаев их применение очень полезно. Например, с их помощью получается естественное доказательство классической теоремы Пуанкаре-Биркгофа-Витта о базисе в универсальной обертывающей алгебре алгебры Ли. Применив технику стандартных базисов, В.Н. Латышев доказал, что в универсальных обертывающих алгебрах нет PI-подалгебр, отличных от коммутативных. Книга В. Н. Латышева, посвященная стандартным базисам в алгебрах, стала популярным учебником.

В работах В.Н. Латышева последнего времени соединились эти два направления: теория PI-алгебр и стандартные базисы. В случае поля характеристики 0 любой $T$-идеал порождается своими полилинейными элементами. В.Н.Латышев ввел понятие комбинаторной системы порождающих для множества полилинейных элементов $T$-идеала и связанное с ним понятие стандартного базиса $T$-идеала. Классическая проблема Шпехта переформулируется в более "жестком" варианте: верно ли, что в случае поля характеристики 0 любой $T$-идеал имеет конечный стандартный базис? Эта вариация проблемы Шпехта в настоящее время остается открытой. В. Н. Латышев привел ее положительное решение в ряде частных случаев - для многообразия, порожденного верхнетреугольными матрицами порядка $n$ (оно задается указанным выше тождеством - произведение $n$ коммутаторов равно нулю), а также для метабелева многообразия, заданного тождеством $\left[\left[x_{1}, x_{2}\right],\left[x_{3}, x_{4}\right]\right]=0$.

Виктор Николаевич в шутку любил повторять, что математики должны заботиться о сохранении своей окружающей среды: "вырубив дерево, надо посадить новое", т.е., решив какую-либо знаменитую проблему, математик обязан сформулировать другую, не менее интересную. Помимо усиленной формы проблемы Шпехта, В.Н. Латышев на семинаре по теории колец в МГУ сформулировал еще одну проблему, которая теперь носит его имя: верно ли, что любая конечно определенная ниль-алгебра нильпотентна? (Вариант: верно ли, что любая конечно определенная алгебраическая алгебра конечномерна?) Напомним, что в алгебре очень популярны проблемы бернсайдовского типа, когда одни условия конечности влекут другие. В частности, для ассоциативных колец знаменитый пример Голода-Шафаревича дает отрицательный ответ на проблему Куроша: верно ли, что любое конечно порожденное ниль-кольцо нильпотентно? Аналогичные контрпримеры были построены в группах (результаты П. С. Новикова и С. И. Адяна, пример А. Ю. Ольшанского - конечно порожденная периодическая группа не обязана быть конечной, даже если порядки всех 
ее элементов ограничены) и других алгебраических структурах. Однако В. Н. Латышев отметил, что во всех построенных контрпримерах конечно порожденных колец и алгебр множество определяющих соотношений бесконечно: не удается построить конечную систему соотношений, обеспечивающую только свойство "ниль" (любой элемент кольца в некоторой степени равен нулю) при отсутствии нильпотентности (любое произведение $n$ элементов равно нулю) - всякая конечная система определяющих соотношений оказывается слишком "грубой" и вместе со свойством "ниль" обеспечивает сразу и нильпотентность. Эта проблема вошла в Днестровскую тетрадь (“Нерешенные проблемы теории колец и модулей”, ИМ СО РАН, 1993, проблема 1.92). Проблема Латышева не решена, причем продвижения в положительном направлении даже в простейших частных случаях невелики (можно ограничивать число переменных, степень соотношений, рассматривать только однородные соотношения и т. п.).

Стиль математических работ В. Н. Латышева отличается ясностью и изяществом, в его статьях не бывает непонятных или сомнительных мест. Зачастую В.Н. Латышев приводил короткие и изящные доказательства уже известных утверждений, полученных другими авторами с помощью тяжелой техники и трудных для восприятия. Яркий пример - теорема Регева о коразмерностях $T$-идеала и следствие из нее, решающее положительно проблему Джекобсона: тензорное произведение двух PI-алгебр также является PI-алгеброй. В.Н. Латышев привел изящное доказательство этих двух теорем, основанное на простом комбинаторном соображении, причем полный текст его статьи занимает всего полторы страницы. Именно это его доказательство вошло в разные монографии, посвященные теории PI-алгебр.

В. Н. Латышев был замечательным педагогом и организатором науки. Долгое время он вместе с Л. А. Скорняковым и А. В. Михалевым руководил работой семинара по теории колец на мехмате МГУ, в котором участвовали студенты, аспиранты и сотрудники как мехмата, так и других вузов и организаций. (По теории колец и модулей было два семинара, на первом из них рассматривались более общие вопросы, второй, “пятничный", был в большей степени посвящен тематике, связанной с PI-алгебрами и комбинаторной теорией.) Постоянными участниками семинара по пятницам были как ученики Виктора Николаевича (С. А. Пихтильков, В.Е. Барбаумов, Т. Гатева, В. В. Борисенко, С.В. Охитин, Н. К. Иыуду, Е. В. Лукоянова, В. В. Щиголев), так и многие другие молодые математики, ученики А.В. Михалева, Л.А. Скорнякова и С. В. Пчелинцева, специализирующиеся в теории колец и модулей (К. И. Бейдар, В. Т. Марков, А. А. Туганбаев, А. В. Гришин, А. Я. Белов и др.); на всех них В. Н. Латышев оказал сильное влияние, заинтересовав тематикой, связанной с РІ-кольцами, комбинаторными и алгоритмическими вопросами теории колец. Среди результатов, полученных участниками семинара, можно отметить описание В. Т. Марковым локально нётеровых и локально представимых многообразий алгебр (этот результат потом был усилен А. З. Ананьиным), описание С. А. Пихтильковым общих тождеств всех $n$-мерных алгебр при $n \leqslant 15$ и его работы по специальным алгебрам Ли (их обертывающая является ассоциативной РІ-алгеброй), результаты В. Е. Барбаумова об автоморфизмах в PI-алгебрах, введение и использование понятия существенной высоты PI-алгебры А. Я. Беловым, структурные и алгоритмические вопросы для мономиальных алгебр (в обзорной статье В.Н.Латышева, А. Я. Белова, В. В. Борисенко), связь между слабыми тождествами, центральными полиномами и свойством устойчивости для $T$-идеалов в работах С. В. Охитина, построение контрпримеров к проблеме Шпехта над полем конечной характеристики А. Я. Беловым, А. В. Гришиным 
и В.В. Щиголевым, алгоритмические вопросы в алгебрах со стандартными базисами в работах Н. К. Иыуду, А. Я. Белова и Е. В. Лукояновой. А. В. Михалев, К. И. Бейдар, М. А. Чеботарь развивали направления, обобщающие теорию PI-алгебр: теорию обобщенных тождеств и GPI-колец, теорию функциональных тождеств. Под руководством В.Н. Латышева было защищено более 25 кандидатских диссертаций, трое его учеников защитили докторские диссертации.

Помимо руководства семинаром, В.Н. Латышев читал спецкурсы по теории ассоциативных и лиевых алгебр. В последние годы в МГУ очень популярен был спецкурс В. Н. Латышева, посвященный эффективным вычислениям в алгебре и различным алгоритмическим аспектам символьных вычислений, а также вопросам сложности алгоритмов. Центральным инструментом в этом курсе были стандартные базисы (базисы Грёбнера-Ширшова). Кроме книги по стандартным базисам перу В. Н. Латышева принадлежат также два замечательных учебных пособия, напрямую, казалось бы, не связанных с областью его научных интересов: пособие, посвященное симплекс-методу и выпуклому программированию, и книжка, посвященная понятию тензорного ранга, обобщающему понятие ранга матрицы. Эти три книжки, опубликованные издательством МГУ, быстро стали библиографической редкостью. Как и все статьи В. Н. Латышева, они написаны в ясном и изящном стиле, не оставляющем места непониманию и сомнениям, что очень ценно для учебной литературы.

Всего В.Н. Латышевым опубликовано 79 научных работ, 10 книг и учебных пособий.

Следует отметить и организационную работу В.Н. Латышева на мехмате МГУ и в филиале МГУ в г. Ульяновске. В течение долгого времени В.Н. Латышев руководил заочным отделением мехмата, а также возглавлял аспирантуру отделения математики мехмата МГУ. Ряд лет он был заместителем заведующего кафедрой алгебры члена-корреспондента РАН А. И. Кострикина, с которым они очень дружили. С 2001 по 2015 г. В. Н. Латышев заведовал кафедрой высшей алгебры. Как руководитель В. Н. Латышев был очень ответственным и при этом совершенно неконфликтным человеком - все, кому он помогал в решении различных вопросов, вспоминают его исключительно добрыми словами. Отметим его роль в создании филиала МГУ в г. Ульяновске, который был организован в 1988 г. (с 1995 г. он приобрел статус самостоятельного университета). В первые годы занятия в нем частично проводили преподаватели из МГУ, ездившие туда в командировки. Но довольно быстро там сложился самостоятельный коллектив, состоящий из высококлассных ученых. В. Н. Латышев был командирован на три года в Ульяновск, где он исполнял обязанности проректора филиала по науке и декана механико-математического факультета филиала МГУ, читал там лекции и вел семинары, а также помогал в решении множества организационных вопросов. Именно В. Н. Латышев сформировал в Ульяновском университете сильный математический коллектив, пригласив таких замечательных математиков, как А.Р. Кемер, С.П. Мищенко, С. В. Охитин, В. М. Петроградский, А. Я. Веревкин и др. Сейчас Ульяновский государственный университет стал одним из ведущих математических центров России.

В.Н. Латышев был награжден грамотой ВАК за заслуги в работе по аттестации научных кадров, грамотой Верховного Совета РФ и медалью “За доблестный труд”, нагрудным знаком "За отличные успехи в работе" Высшей школы СССР, медалью ВДНХ. В 2001 г. В.Н. Латышеву было присуждено звание "Заслуженный профессор Московского университета". В 2006 г. В.Н. Латышев стал лауреатом премии им. М. В. Ломоносова за педагогическую деятельность. 
Все, кто знал Виктора Николаевича Латышева, будут помнить его как замечательного математика, прекрасного педагога, организатора науки и просто очень хорошего человека.

Список научных трудов В. Н. Латышева и другую информацию о нем можно найти в системе "Истина" МГУ: https://istina.msu.ru/profile/latyshev/.

В.А. Артамонов, Л.А. Бокуть, В.В. Борисенко, Е.И. Бунина, В.М. Бухштабер, А. Э. Гутерман, Ю. Л. Ершов, М. В. Зайцев, А. Я. Канель-Белов, А.Р. Кемер, А.В. Михалев, С.П. Мищенко, С.П. Новиков, А.Ю. Ольшанский, Ю.Г. Прохоров, Ю.П. Размыслов, А.И. Шафаревич, И.В. Шестаков 